\title{
Diagnostic Value of Systemic Cholesteryl Ester/Free Cholesterol Ratio in Hepatocellular Carcinoma
}

\author{
SABRINA KRAUTBAUER ${ }^{1,2}$, THOMAS S. WEISS ${ }^{3}$, REINER WIEST $^{4}$, DORIS SCHACHERER $^{1}$, \\ GERHARD LIEBISCH${ }^{2}$ and CHRISTA BUECHLER ${ }^{1}$ \\ ${ }^{1}$ Department of Internal Medicine I, Regensburg University Hospital, Regensburg, Germany; \\ ${ }^{2}$ Institute of Clinical Chemistry and Laboratory Medicine, Regensburg University Hospital, Regensburg, Germany; \\ ${ }^{3}$ University Children Hospital (KUNO), Regensburg University Hospital, Regensburg, Germany; \\ ${ }^{4}$ Department of Visceral Surgery and Medicine, University Inselspital, Bern, Switzerland
}

\begin{abstract}
Background: Hepatocellular carcinoma (HCC) most commonly occurs in the setting of liver cirrhosis which is characterized by low serum lipids. We hypothesized that composition of lipoproteins and consequently lipid species ratios are mostly unchanged in patients with cirrhosis compared to controls. This approach may be appropriate to identify lipid ratios altered in HCC irrespective of liver dysfunction. Patients and Methods: Lipids were measured in serum of 21 patients with HCC, 41 patients with liver cirrhosis and 22 controls. Ratios of lipids known to be changed in HCC tissues were calculated. Results: Ratios of polyunsaturated to mono-unsaturated lysophosphatidylcholine, ceramidel sphingomyelin and cholesteryl ester/free cholesterol were changed in HCC compared to both control cohorts. The latter was most suited to diagnosing HCC. Systemic ratios of these lipid classes were not associated with fibrosis, staging or grade in patients with HCC. Conclusion: The cholesteryl ester/free cholesterol ratio is comparable in controls and patients with cirrhosis, but is specifically increased in patients with HCC.
\end{abstract}

Viral infections, excessive alcohol intake, and non-alcoholic fatty liver disease (NAFLD) are the main pathologies of liver cirrhosis. Hepatocellular carcinoma (HCC) may arise in cirrhotic liver, but also occurs in non-cirrhotic NAFLD. The pathophysiology of non-cirrhotic HCC has not been clarified and surrogate markers to identify patients at risk for $\mathrm{HCC}$ development are not available. Patients with NALFD without

Correspondence to: Christa Buechler, Ph.D., Department of Internal Medicine I, Regensburg University Hospital, D-93042 Regensburg, Germany. Tel: +49 9419447009, Fax: +49 9419447019, e-mail: christa.buechler@klinik.uni-regensburg.de

Key Words: Phospholipids, liver cirrhosis, cholesterol, hepatocellular carcinoma. cirrhosis are not screened regularly for $\mathrm{HCC}$ and may, therefore, be diagnosed with advanced-stage $\operatorname{HCC}(1,2)$.

Chronic liver dysfunction is related to disturbed lipid metabolism, and consequently systemic lipid species are changed (3-5). Mass spectrometric techniques enable the measurement of various lipid species (6-9). Serum lipids can be easily analyzed and may evolve as non-invasive biomarkers for various diseases. A challenge is the identification of circulating lipids which are specifically changed in patients in order to establish biomarkers for liver fibrosis or HCC $(3-5,10,11)$.

Approaches to identifying systemic lipids suitable as biomarkers for $\mathrm{HCC}$ need to consider that the serum lipidome is markedly altered in patients with liver cirrhosis when compared to suitable controls. Indeed, various lipids are comparably changed in patients with liver cirrhosis and those suffering from $\operatorname{HCC}(4,5,10)$. Identification of appropriate lipid biomarkers is further challenged by HCC arising in non-cirrhotic liver in those with NAFLD (1).

Lysophosphatidylcholine (LPC) is diminished in serum of patients with NAFLD and is negatively associated with Model of End-stage Liver Disease (MELD) score in cirrhosis $(11,12)$. Reduced LPC levels have been described in patients infected with chronic hepatitis B with liver cirrhosis. In serum of patients with HCC of different disease etiologies, concentrations of distinct LPC species are further reduced (4, 5). A combination of three metabolites, with one of them being LPC 22:5, was found to discriminate patients with HCC from those without HCC with a higher sensitivity and specificity than alpha-fetoprotein (13).

The sphingolipid ceramide induces apoptotic cell death, and therefore, is regarded to act as a tumor suppressor (14). Ceramide levels are indeed diminished in tumors and are markedly lower in HCC tissues $(14,15)$. Long-chain ceramides (C16-C20) induce apoptosis, while very longchain ceramides (C22-C24) exert the opposite effect and even promote cell proliferation (16). This shows that 
Table I. Characteristics of the patient cohorts (median values and range).

\begin{tabular}{lcccc}
\hline & HCC & Cirrhosis & Controls & $p$-Value \\
\hline Males/females, $\mathrm{n}$ & $21 / 0$ & $32 / 9$ & $8 / 14$ & $0.005^{\# \S}$ \\
Age, years & $63(47-84)$ & $54(26-81)$ & $53(21-88)$ & $0.012^{\#}, 0.041^{\S}$ \\
BMI, $\mathrm{kg} / \mathrm{m}^{2}$ & $28(20-45)$ & $26.0(16.0-38.0)$ & $25(18.0-38)$ & $0.01^{\#}$ \\
T2D, $\mathrm{n}$ & 11 & 12 & 3 & $20(12-40)$ \\
ALT, U/l & $42(23-378)^{\mathrm{a}}$ & $29(2-84)$ & $26(16-48)$ & $0.003^{\#}, 0.020^{\S}$ \\
AST, U/l & $32(14-145)^{\mathrm{a}}$ & $37(4-108)$ & $0.5(0.3-1.9)$ & $<0.001^{*}, 0.002^{\S}$ \\
Bilirubin mg/dl & $0.6(0.2-2.5)^{\mathrm{a}}$ & $1.3(0.3-8.2)$ &
\end{tabular}

ALT: Alanine aminotransferase; AST: aspartate aminotransferase; BMI: body mass index; T2D: type 2 diabetes. Significant differences: *Controls $v s$. patients with liver cirrhosis, ${ }^{\#}$ controls $v s$. patients with HCC, ${ }^{\S}$ patients with liver cirrhosis $v s$. patients with HCC. aData available only for 20 patients.

disequilibrium between ceramides with different acyl chains and not changes of individual species may be relevant for tumor growth (16). Analysis of systemic ceramides in patients with HCC of different etiologies revealed higher levels of long- and very long-chain ceramides (10). Whether the ratio of these lipid classes is different from patients with liver cirrhosis, who served as controls, has not been considered (10). As well as sphingolipids, cholesterol and phospholipid species are also changed in HCC tissues $(17,18)$.

A recently performed analysis of lipid species by our group using paired samples of HCC tissues and adjacent non-tumorous tissues demonstrated that levels of various lipid species were altered in the tumors. Of note, ceramides were reduced and sphingomyelins (SMs) were increased in HCC tissue. Regarding phospholipids, we found raised levels of saturated and lower amounts of polyunsaturated species (PUFA) (15). Although not shown in our previous study (15), the quotients of the related lipid classes are obviously prominently changed especially when compared to the individual lipid classes.

This led us to hypothesize that the ratios of related lipid species may be altered in serum of patients with HCC. Considering that low systemic lipid levels are a feature of liver cirrhosis, calculation of ratios minimizes the identification of lipids simply related to dyslipidemia. Therefore, we measured different lipids in serum of patients with HCC, liver cirrhosis, and controls, and calculated the ratios of lipids identified to be differentially abundant in HCC (15).

\section{Materials and Methods}

Patients. The patients with HCC included here were described in a previous study (15). Experimental procedures were performed according to the guidelines of the charitable state-controlled foundation Human Tissue and Cell Research (19).
Serum of patients with liver cirrhosis used here was used in previous studies including (11). Etiology of liver cirrhosis was alcoholic in 35 , hepatitis $\mathrm{C}$ infection in three, and other reasons in three patients. Controls were randomly selected from a cohort of patients referred for ultrasound examination of the abdomen and patients without fatty liver (20). The study was approved by the local Ethical Committee of the University Hospital of Regensburg (approval number 15-101-0318) and written informed patient consent was obtained. The study groups are described in Table I.

Quantification of lipids. Quantification of lipid species was carried out by direct flow injection electrospray ionization tandem mass spectrometry (ESI-MS/MS) in positive ion mode as described previously (9). Non-naturally-occurring lipid species served as internal standards. Analysis was performed on a Quattro Ultima triple-quadrupole mass spectrometer (Micromass, Manchester, UK) equipped with a binary pump (Model 1100; Agilent, Waldbronn, Germany) and an autosampler (HTS PAL, Zwingen, Switzerland). For phosphatidylcholine (PC), SM and lysophosphatidylcholine (LPC) a fragment ion of $\mathrm{m} / \mathrm{z}, 184$ was used $(7,9)$. A neutral loss of $141 \mathrm{Da}$ was used for phosphatidylethanolamine (PE) and of $277 \mathrm{Da}$ for phosphatidylinositol (PI) (21). For phosphatidylserine (PS) a neutral loss scan of $\mathrm{m} / \mathrm{z} 185$ was used. Sphingosine-based ceramides and SM annotations assume presence of d18:1. Ceramides were determined using a fragment ion of $m / z, 264$ (22). Free cholesterol (FC), selectively derivatized, and cholesteryl ester (CE) were analyzed by a fragment ion of $m / z 369$ (6). Correction of lipid species isotopic overlap and data analysis were performed by selfprogrammed Excel Macros. Lipid species annotation was according to the published proposal for short-hand notation (23). Glycerophospholipid annotation assumes even-numbered carbon chains only.

Statistical analysis. Data are shown as box plots indicating median, lower and upper quartiles and range of the values. Statistical analysis was carried out using ANOVA with post-hoc Bonferroni correction, Mann-Whitney $U$-test, Receiver operating characteristics curve or Spearman correlation (SPSS Statistics 21.0; IBM Corp., Armonk, NY, USA). In order to account for multiple comparisons in correlation analysis, $p$-values were multiplied by 5 . Youden's index was calculated as: Sensitivity + Specificity -1 . Values of $p<0.05$ were regarded as significant. 


\section{Results}

Study cohort. Lipid species were analyzed in serum of 21 patients with HCC, 41 patients with liver cirrhosis and 22 controls (Table I). Measurements of lipid species in sera of patients were performed within one batch to avoid interassay variations. It is important to note that nine of the patients with HCC did not have liver fibrosis.

Phospholipid ratios. Impaired hepatic function in liver cirrhosis is associated with reduced levels of lipoproteins (24). These particles are composed of triglycerides, cholesterol, LPC, PC, PE, SM and ceramide (25). Therefore, changes of the absolute levels of lipid species may mostly be related to dyslipidemia in liver cirrhosis, while composition of lipoproteins, and consequently ratios of individual lipids or lipid classes may be unaltered (26).

Our recent study revealed reduced levels of PUFAcontaining phospholipids and higher concentrations of saturated fatty acid (SFA)-containing phospholipids in HCC tissues when compared to non-tumorous liver (15).

Ratios of PUFA/SFA PE and PI in serum were, however, not different between the groups (data not shown). LPC PUFA/SFA ratios were significantly lower in cirrhosis and HCC compared to controls but were similar in HCC and cirrhosis (Figure 1A). The PC PUFA/SFA ratio was lowest in patients with liver cirrhosis. There was no difference between HCC and controls (Figure 1B). Only PC PUFA/SFA ratio negatively correlated with MELD score in patients with liver cirrhosis (Figure $1 \mathrm{C}$ and data not shown). Although MELD score for patients with HCC was not calculated, nine patients did not have liver fibrosis, indicating that the median MELD score was lower in this cohort. Thus, PC PUFA/SFA ratio appears to be associated with residual liver function.

Levels of mono-unsaturated fatty acids (MUFA) in PC and PE were recently identified to be higher in human HCC tissues (17). Therefore, PUFA/MUFA ratios of the phospholipids measured were also calculated. PE PUFA/MUFA was highest in the controls but not different between patients with cirrhosis and those with HCC (Figure 1D). PI PUFA/MUFA was similar in patients with HCC and those with cirrhosis, with the latter displaying lower levels than those of the controls (Figure 1E). LPC PUFA/MUFA was reduced in patients with HCC and those with cirrhosis compared to controls, and was in fact lowest in cirrhosis (Figure 1F). PC PUFA/MUFA was specifically reduced in cirrhosis (Figure $1 \mathrm{G}$ ).

A decreased $\mathrm{PC} / \mathrm{PE}$ ratio in the liver is linked to hepatocyte ballooning and inflammation (27). While analyzing our samples, we did not find any difference in the $\mathrm{PC} / \mathrm{PE}$ ratio in the three cohorts (Figure $1 \mathrm{H}$ ).

Cholesterol ratios. Cholesterol production is induced in HCC tissue and higher serum cholesterol has been described in patients with HCC $(17,28)$. Total cholesterol was not different between the groups studied herein and FC was even reduced in serum from patients with HCC compared to controls (data not shown), suggesting that the $\mathrm{CE}$ to $\mathrm{FC}$ ratio is increased in HCC. Indeed, this ratio was similar in the control group and patients with liver cirrhosis but was significantly raised in those with HCC (Figure 2A).

Sphingolipid ratios. In HCC tissues, the ceramide level is low and sphingomyelin is increased, indicating lower activity of sphingomyelinase (15). In serum of patients with HCC, long- (C16-C20) and very long-chain (C22-C24) ceramides are even induced, while data on sphingomyelin were not available in this study (10). Ceramide/SM ratio was higher in HCC and similar in the control cohorts and patients with liver cirrhosis (Figure 2B).

Because long- and very long-chain ceramide have opposing functions regarding cell death, this ratio was also calculated. The quotient was highest in patients with liver cirrhosis but similar in controls and those with HCC (Figure 2C). Positive correlation of long-/very long-chain ceramide with MELD score shows that this ratio is associated with the extent of liver injury (Figure 2D).

Correlations with age, body mass index, bilirubin and aminotransferases. Correlations of lipid ratios with age, body mass index, bilirubin and aminotransferases were calculated separately for the three groups. Here, PC PUFA/SFA ratio $(\mathrm{r}=-0.535, p<0.001)$ and PC PUFA/MUFA ratio $(\mathrm{r}=-0.505, p=0.005)$ were negatively correlated with serum bilirubin in the cohort of patients with liver cirrhosis.

Lipid analysis in males. Females were not included in the HCC cohort study. However, LPC PUFA/SFA, LPC PUFA/MUFA, ceramide/SM and CE/FC ratios did not differ in the 14 female and the eight male controls (Figure 2E and F) and data not shown). The nine female and the 32 male patients with cirrhosis had similar ratios of these lipid classes (data not shown).

In order to further exclude whether HCC-associated changes were related to gender, calculations were carried out using data obtained from serum from males only. In principle, results were comparable to the distribution seen for the whole cohort. Ceramide/SM and $\mathrm{CE} / \mathrm{FC}$ ratios were higher in male patients with HCC compared to patients with cirrhosis and controls (Figure 3A and B). LPC PUFA/SFA ratio steadily declined from controls to cirrhosis to HCC and was significantly lower in HCC compared to controls (Figure 3C). LPC PUFA/MUFA was lowest in patients with cirrhosis but was also reduced in patients with $\mathrm{HCC}$ compared to controls (Figure 3D). Receiver operating characteristic curve analysis of lipid ratios to detect HCC in the whole cohort revealed an area under the curve (AUC) 

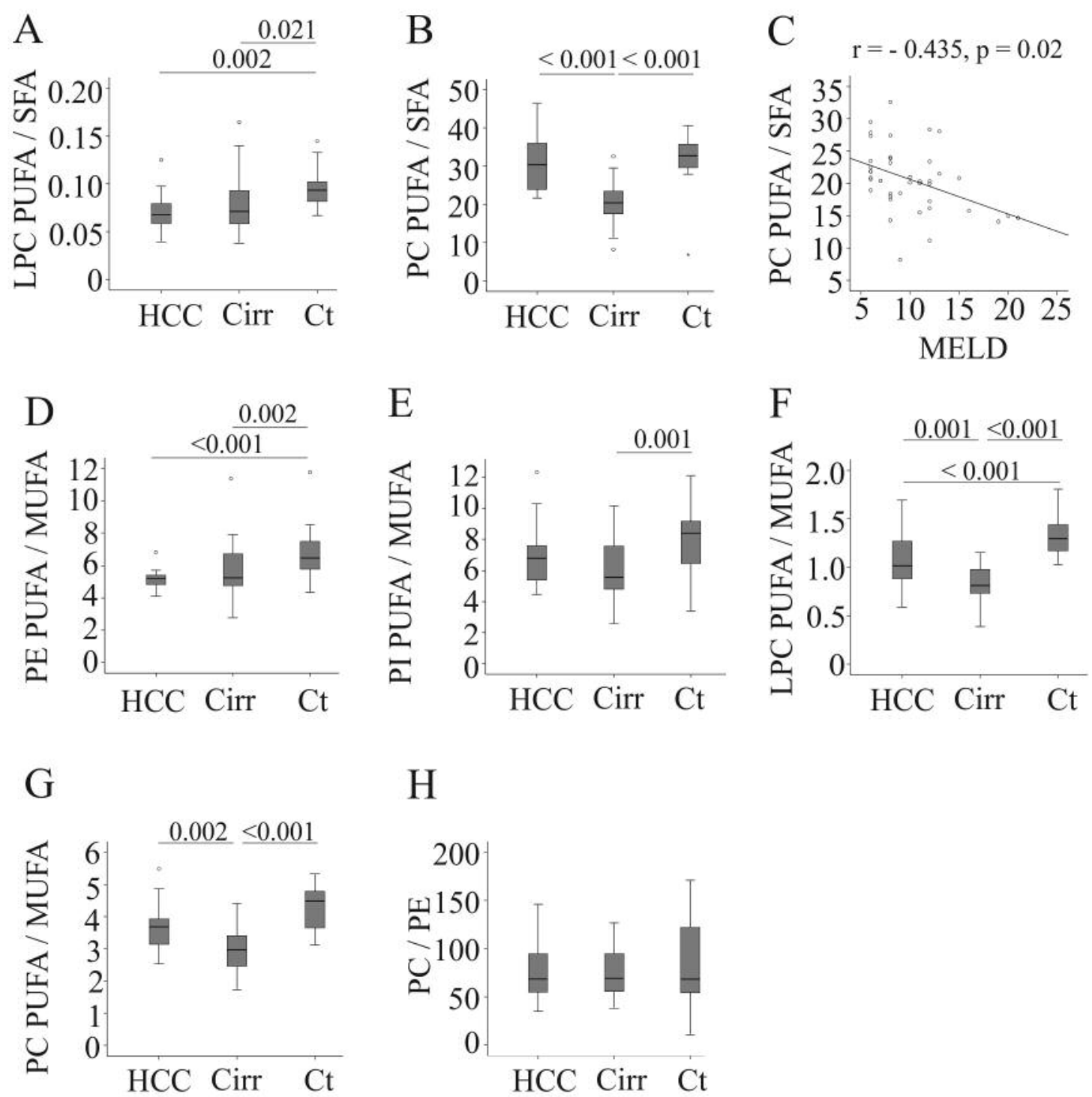

Figure 1. Phospholipid ratios are not specifically changed in patients with hepatocellular carcinoma (HCC). Polyunsaturated fatty acid $(P U F A)$ /saturated fatty acid (SFA) lysophosphatidylcholine (LPC) (A) and PUFA/SFA phosphatidylcholine (PC) (B) in patients with HCC, with liver cirrhosis (Cirr) and controls (Ct). Correlation of PC PUFA/SFA ratio with Model of End-Stage Liver Disease (MELD) score in patients with liver cirrhosis (C). PUFA/mono-unsaturated fatty acid (MUFA) phosphatidylethanolamine (PE) (D), PUFA/MUFA phosphatidylinositol (PI) (E), PUFA/MUFA LPC (F), PUFA/MUFA PC $(G)$ and PC/PE (H) in patients with HCC, with liver cirrhosis (Cirr) and in controls (Ct). Median, lower and upper quartiles and range of the values are shown. p-Values for differences and correlations are given.

of 0.543 for LPC PUFA/MUFA, of 0.746 for ceramide/ sphingomyelin and of 0.866 for $\mathrm{CE} / \mathrm{FC}$ ratio (Figure 4A). In the male group, the corresponding AUCs were $0.606,0.774$ and 0.878 , respectively (Figure 4B). This shows that the $\mathrm{CE} / \mathrm{FC}$ ratio is best suited to $\mathrm{HCC}$ diagnosis. The optimal cut-off value for the $\mathrm{CE} / \mathrm{FC}$ ratio for the whole cohort was 2.46 and identified $\mathrm{HCC}$ with a sensitivity of $76.2 \%$ and a specificity of $90.3 \%$.
Association with staging, differentiation grade and fibrosis in $H C C$. In the 21 patients with HCC, neither systemic ceramide/SM nor LPC PUFA/MUFA or CE/FC ratios were related to T-stage or differentiation grade (Figure 5A-D and data not shown).

Of the 21 patients with $\mathrm{HCC}, 9$ did not display liver fibrosis. Ceramide/SM, LPC PUFA/MUFA and CE/FC ratios were comparable in these groups, showing that altered levels are not related to liver fibrosis (Figure $5 \mathrm{E}$ and $\mathrm{F}$, and data not shown). 

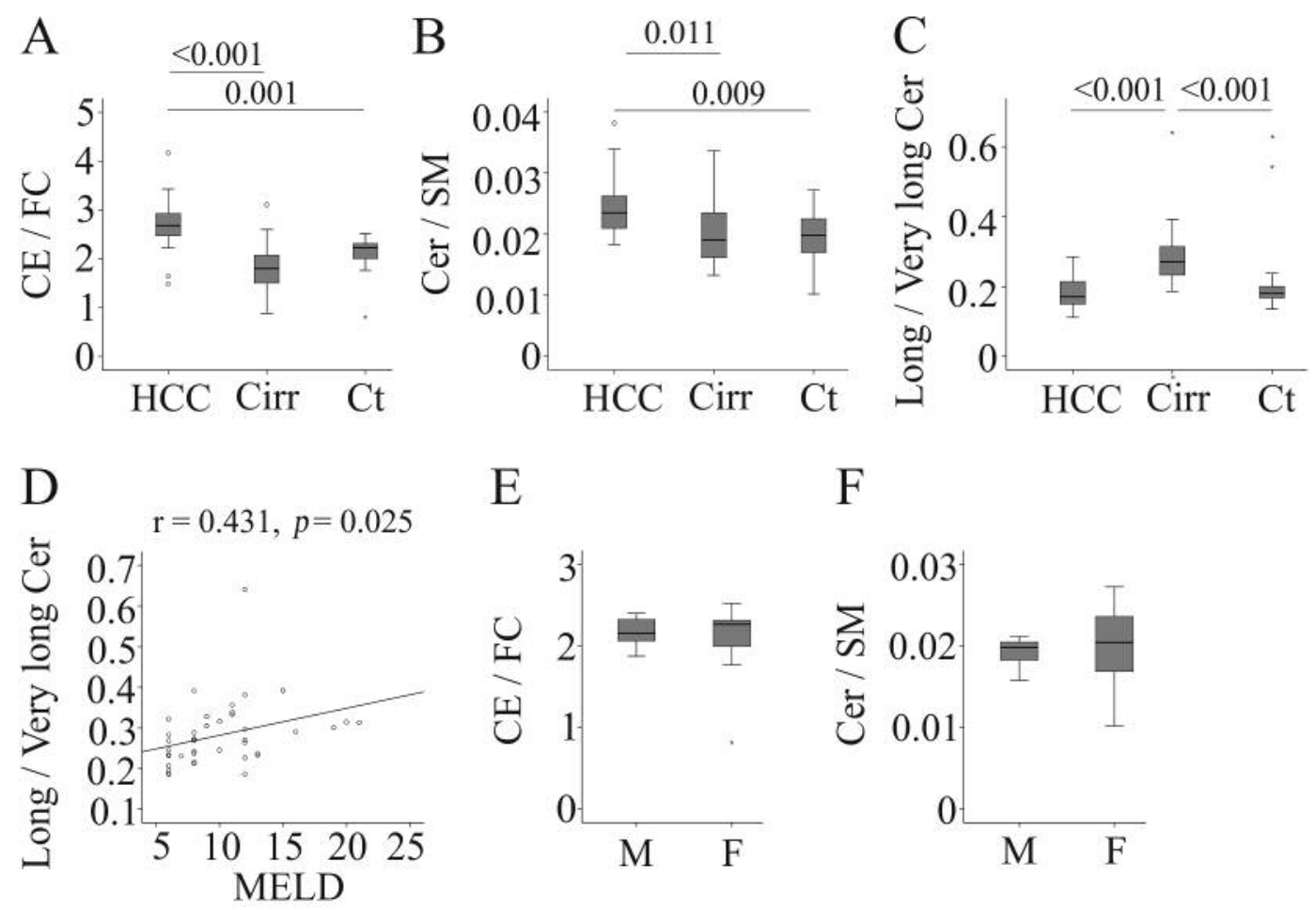

Figure 2. Cholesterol and sphingolipid ratios are similar in both gender and are increased in patients with hepatocellular carcinoma (HCC). A: Cholesteryl ester (CE)/free cholesterol (FC), B: ceramide (Cer)/sphingomyelin (SM) and C: long- (C16-C20)/very long-(C22-C24) chain ceramide in patients with HCC, with liver cirrhosis (Cirr) and controls (Ct). D: Correlation of long-/very long-chain ceramide ratio with Model of End-Stage Liver Disease (MELD) score in patients with liver cirrhosis. E: CE/FC ratio in male $(M)$ and female $(F)$ controls $(C t)$. F: Cer/SM ratio in male $(M)$ and female $(F)$ controls. Median, lower and upper quartiles and range of the values are shown. p-Values for differences and correlations are given.

\section{Discussion}

This analysis revealed that $\mathrm{CE} / \mathrm{FC}$ ratio is increased in serum from patients with HCC when compared to controls and patients with liver cirrhosis. The LPC PUFA/MUFA ratio was found to be lowest in cirrhosis, but also reduced in patients with HCC when compared to controls. The ceramide/SM ratio was highest in $\mathrm{HCC}$, but levels did not significantly differ from those of controls when calculations were carried out for males only. Although this does not exclude the possibility that the ceramide/SM ratio is indeed elevated in patients with $\mathrm{HCC}$, changes in the $\mathrm{CE} / \mathrm{FC}$ quotient were found to be most pronounced. Receiver operation characteristic curve analysis revealed that this ratio is best suited to diagnosing HCC.

CE was found to be modestly higher in HCC tissues compared to adjacent non-tumorous tissues, while FC was not changed, suggesting that their ratio is increased in the tumor (15). Cellular cholesterol synthesis is enhanced in liver cancer cells, and sterol regulatory element-binding transcription factor 2 and low-density lipoprotein receptor expression are induced (29). Higher endogenous cholesterol biosynthesis and uptake of low-density lipoprotein particles are essential for cell proliferation and survival (30). Blocking acyl-CoA cholesterol acyltransferase-1 suppresses proliferation and induces apoptosis of cancer cells, and this is attributed to the cytotoxic effects of free cholesterol (31). Although a higher $\mathrm{CE} / \mathrm{FC}$ ratio is therefore supposed to promote cancer growth (31), it was not found to be changed with differentiation grade and $\mathrm{T}$-stage in the present cohort. The number of patients enrolled was, however, very small and further studies are needed to exclude any such association. An increased $\mathrm{CE} / \mathrm{FC}$ ratio in HCC may possibly be used as a biomarker to diagnose HCC in asymptomatic patients.

The lipid profile of patients with liver cirrhosis and HCC is highly similar (32). Compared to healthy controls most of lipid levels are low in patients with liver cirrhosis and HCC (32). Negative associations of blood total cholesterol levels and MELD score have been described (33). Therefore, differences in lipid concentrations mostly reflect the degree of liver dysfunction rather than being specifically changed in patients with HCC.

This does not apply to PC PUFA/SFA and long-/very long-chain ceramide ratios which are specifically altered in 
A
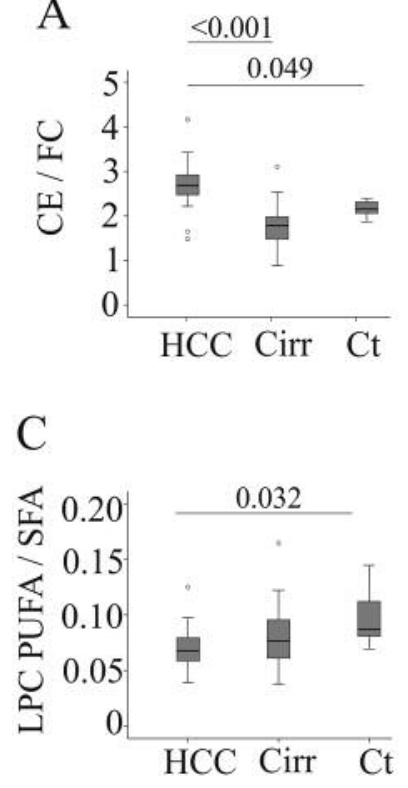

B

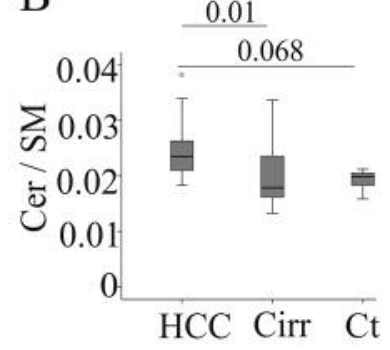

D

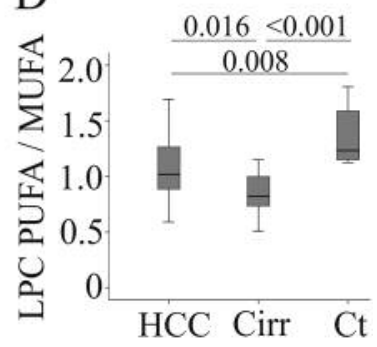

A

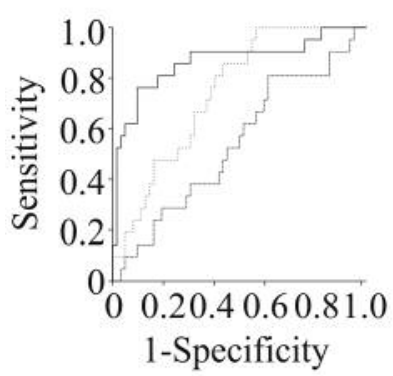

B

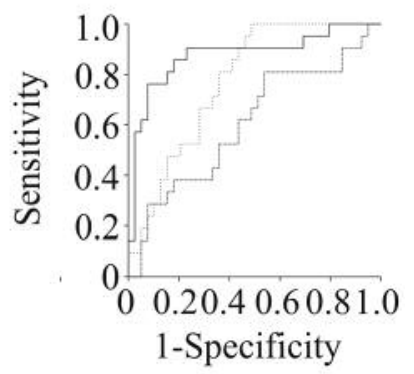

Figure 3. Lipid ratios in males are similarly changed as in the whole cohort. Cholesteryl ester (CE)/free cholesterol (FC) (A), ceramide (Cer)/sphingomyelin (SM) (B), polyunsaturated fatty acid (PUFA)/saturated fatty acid (SFA) lysophosphatidylcholine (LPC) ratio (C) and PUFA/mono-unsaturated fatty acid (MUFA) LPC (D) in male patients with hepatocellular carcinoma (HCC), with liver cirrhosis (Cirr) and controls $(C t)$. Median, lower and upper quartiles and range of the values are shown. p-Values for differences and correlations are given.

patients with liver cirrhosis. Both of these ratios correlate with MELD score, demonstrating that the distribution of these lipid classes is associated with hepatic dysfunction. Thus, equilibrium of distinct lipid classes is obviously changed in liver cirrhosis and further studies are needed to evaluate whether this is of any pathophysiological or diagnostic value.

A further issue is the identification of HCC in the noncirrhotic liver of patients with NAFLD. Such patients more often have dyslipidemia and an elevated serum cholesterol level (34). Hypercholesterolemia has been also described in HCC with steato-hepatitic features (35). This suggests that most lipids are concordantly changed in patients with dyslipidemias and therefore the ratios of different lipids should be mostly unaffected.

Recently, we showed reduced levels of PUFA phospholipids and induced concentrations of saturated phospholipid species in HCC tissues (15). An independent study described that levels of MUFA phospholipids are higher in HCC tissue compared to the adjacent liver (17). PUFA phospholipid to saturated phospholipid quotient is also lower in patients with alcoholic liver cirrhosis when compared to healthy controls (36). Indeed, the respective

LPC and PC PUFA/SFA ratios and PUFA/MUFA ratios are significantly reduced in the patients with liver cirrhosis compared to controls. This does not apply to PE and PI PUFA/SFA ratios, which are comparable in the three groups. Of note, only the LPC PUFA/MUFA ratio is specifically changed in serum of patients with HCC. Lower levels of LPC have been identified in serum from patients with HCC but the respective ratios have not been calculated (37). Receiver operating characteristic curve analysis revealed a low AUC for the LPC PUFA/MUFA ratio, which is therefore unsuitable as a non-invasive marker for HCC diagnosis.

A recent study showed higher levels of long- and very long-chain ceramides in the serum of patients with HCC as compared to patients with cirrhosis, while data on SMs were not provided (10). In that study, patients with HCC had a significantly lower MELD score and concordantly, fewer patients had Child-Pugh C cirrhosis (10). Considering that serum long- and very-long chain ceramides decrease with increasing severity of liver cirrhosis (3), a higher ceramide level in patients with HCC (10) may at least in part reflect less severe liver injury.

The ceramide/SM ratio was similar in controls and patients with liver cirrhosis in the present cohort, indicating that both lipid classes are similarly reduced in these patients. The quotient of these two lipid classes nevertheless increased in patients with HCC, showing that the proportion is specifically changed in patients with tumor.

Only the CE/FC quotient was increased in both cancer tissue (15) and serum, while further lipid ratios were not concordantly changed. This excludes the notion that serum 

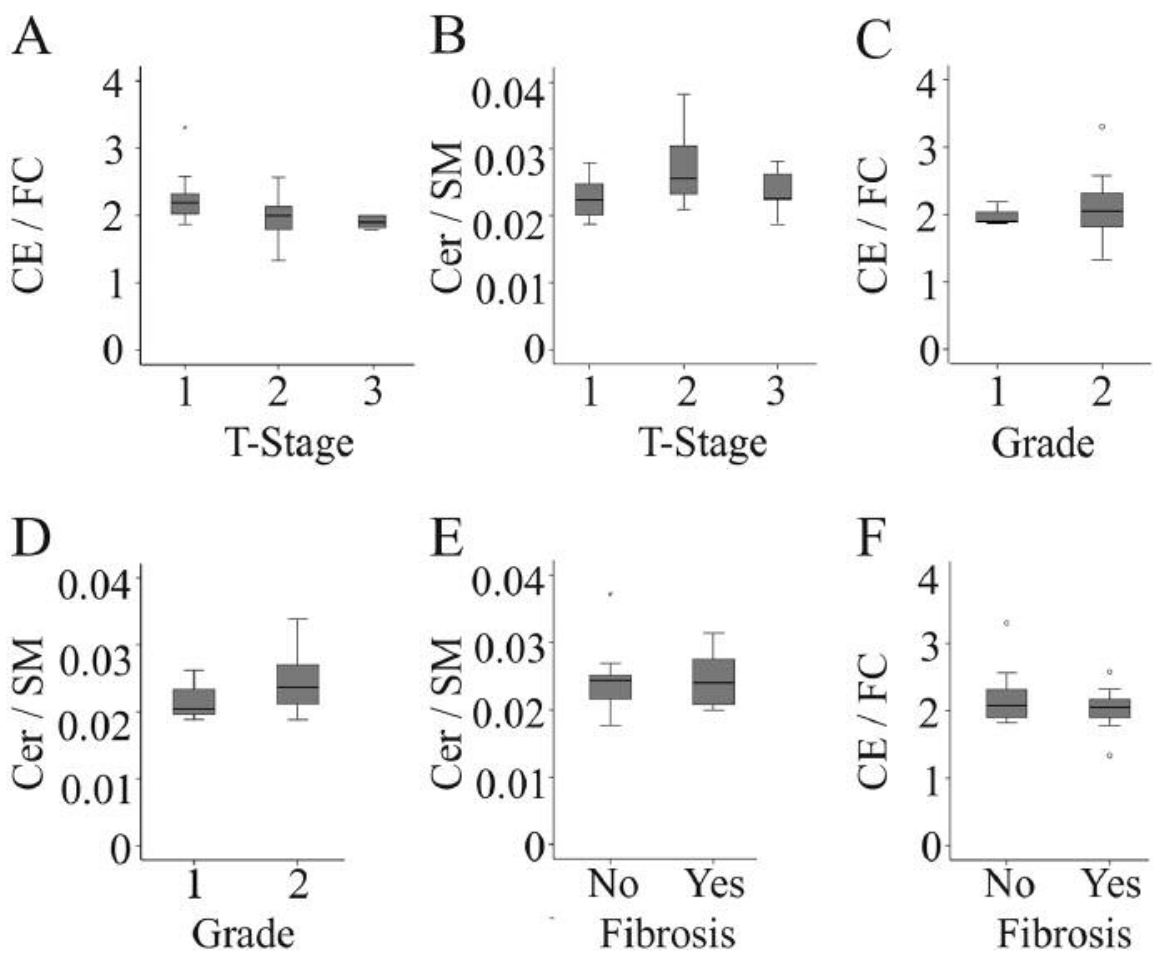

Figure 5. Associations of lipid ratios with T-stage, differentiation grade and fibrosis in patients with hepatocellular carcinoma (HCC). Cholesteryl ester $(C E) /$ free cholesterol $(F C)$ ratio $(A)$ and ceramide (Cer)/sphingomyelin $(S M)$ ratio $(B)$ stratified by $T$-stage. CE/FC ratio $(C)$ and Cer/SM ratio (D) stratified by grade. Cer/SM ratio $(E)$ and CE/FC ratio $(F)$ in patients with HCC without and with liver fibrosis. Median, lower and upper quartiles and range of the values are shown.

lipids specifically associated with $\mathrm{HCC}$ are directly or inversely related to the altered lipidome in liver tumors.

In summary, the present study suggests that the CE/FC ratio is specifically increased in patients with $\mathrm{HCC}$ and may become a useful diagnostic marker.

\section{Acknowledgements}

This study was supported by the Stiftung für Pathobiochemie und Molekulare Diagnostik and partly by the German Research Foundation (BU 1141/13-1). The technical assistance of Jolante Aiwanger, Simone Düchtel and Doreen Müller is greatly appreciated.

\section{References}

1 Kawada N, Imanaka K, Kawaguchi T, Tamai C, Ishihara R, Matsunaga T, Gotoh K, Yamada T and Tomita Y: Hepatocellular carcinoma arising from non-cirrhotic nonalcoholic steatohepatitis. J Gastroenterol 44: 1190-1194, 2009.

2 Marengo A, Rosso C and Bugianesi E: Liver cancer: connections with obesity, fatty liver, and cirrhosis. Annu Rev Med 67: 103$117,2015$.

3 Grammatikos G, Ferreiros N, Waidmann O, Bon D, Schroeter S, Koch A, Herrmann E, Zeuzem S, Kronenberger B and
Pfeilschifter J: Serum sphingolipid variations associate with hepatic decompensation and survival in patients with cirrhosis. PLoS One 10: e0138130, 2015.

4 Patterson AD, Maurhofer O, Beyoglu D, Lanz C, Krausz KW, Pabst T, Gonzalez FJ, Dufour JF and Idle JR: Aberrant lipid metabolism in hepatocellular carcinoma revealed by plasma metabolomics and lipid profiling. Cancer Res 71: 6590-6600, 2011.

5 Zhou L, Ding L, Yin P, Lu X, Wang X, Niu J, Gao P and Xu G: Serum metabolic profiling study of hepatocellular carcinoma infected with hepatitis B or hepatitis $\mathrm{C}$ virus by using liquid chromatography-mass spectrometry. J Proteome Res 11: 54335442, 2012.

6 Liebisch G, Binder M, Schifferer R, Langmann T, Schulz B and Schmitz G: High throughput quantification of cholesterol and cholesteryl ester by electrospray ionization tandem mass spectrometry (ESI-MS/MS). Biochim Biophys Acta 1761: 121$128,2006$.

7 Liebisch G, Drobnik W, Lieser B and Schmitz G: Highthroughput quantification of lysophosphatidylcholine by electrospray ionization tandem mass spectrometry. Clin Chem 48: 2217-2224, 2002.

8 Liebisch G, Drobnik W, Reil M, Trumbach B, Arnecke R, Olgemoller B, Roscher A and Schmitz G: Quantitative measurement of different ceramide species from crude cellular extracts by electrospray ionization tandem mass spectrometry (ESI-MS/MS). J Lipid Res 40: 1539-1546, 1999. 
9 Liebisch G, Lieser B, Rathenberg J, Drobnik W and Schmitz G: High-throughput quantification of phosphatidylcholine and sphingomyelin by electrospray ionization tandem mass spectrometry coupled with isotope correction algorithm. Biochim Biophys Acta 1686: 108-117, 2004.

10 Grammatikos G, Schoell N, Ferreiros N, Bon D, Herrmann E, Farnik H, Koberle V, Piiper A, Zeuzem S, Kronenberger B, Waidmann $\mathrm{O}$ and Pfeilschifter J: Serum sphingolipidomic analyses reveal an upregulation of C16-ceramide and sphingosine-1-phosphate in hepatocellular carcinoma. Oncotarget 7(14): 18095-18105, 2016

11 Krautbauer S, Eisinger K, Wiest R, Liebisch G and Buechler C: Systemic saturated lysophosphatidylcholine is associated with hepatic function in patients with liver cirrhosis. Prostaglandins Other Lipid Mediat 124: 27-33, 2016.

12 Lehmann R, Franken H, Dammeier S, Rosenbaum L, Kantartzis K, Peter A, Zell A, Adam P, Li J, Xu G, Konigsrainer A, Machann J, Schick F, Hrabe de Angelis M, Schwab M, Staiger $\mathrm{H}$, Schleicher E, Gastaldelli A, Fritsche A, Haring HU and Stefan N: Circulating lysophosphatidylcholines are markers of a metabolically benign nonalcoholic fatty liver. Diabetes Care 36: 2331-2338, 2013.

13 Tan Y, Yin P, Tang L, Xing W, Huang Q, Cao D, Zhao X, Wang W, Lu X, Xu Z, Wang H and Xu G: Metabolomics study of stepwise hepatocarcinogenesis from the model rats to patients: potential biomarkers effective for small hepatocellular carcinoma diagnosis. Mol Cell Proteomics 11: M111 010694, 2012.

14 Oskouian B and Saba JD: Cancer treatment strategies targeting sphingolipid metabolism. Adv Exp Med Biol 688: 185-205, 2010 .

15 Krautbauer S, Meier EM, Rein-Fischboeck L, Pohl R, Weiss TS, Sigruener A, Aslanidis C, Liebisch G and Buechler C: Ceramide and polyunsaturated phospholipids are strongly reduced in human hepatocellular carcinoma. Biochim Biophys Acta 1861: 1767-1774, 2016.

16 Hartmann D, Lucks J, Fuchs S, Schiffmann S, Schreiber Y, Ferreiros N, Merkens J, Marschalek R, Geisslinger G and Grosch S: Long-chain ceramides and very long chain ceramides have opposite effects on human breast and colon cancer cell growth. Int J Biochem Cell Biol 44: 620-628, 2012.

17 Abel S, De Kock M, van Schalkwyk DJ, Swanevelder S, Kew MC and Gelderblom WC: Altered lipid profile, oxidative status and hepatitis B virus interactions in human hepatocellular carcinoma. Prostaglandins Leukot Essent Fatty Acids 81: 391399, 2009.

18 Morita Y, Sakaguchi T, Ikegami K, Goto-Inoue N, Hayasaka T, Hang VT, Tanaka H, Harada T, Shibasaki Y, Suzuki A, Fukumoto K, Inaba K, Murakami M, Setou M and Konno H: Lysophosphatidylcholine acyltransferase 1 altered phospholipid composition and regulated hepatoma progression. J Hepatol 59: 292-299, 2013.

19 Thasler WE, Weiss TS, Schillhorn K, Stoll PT, Irrgang B and Jauch KW: Charitable State-Controlled Foundation Human Tissue and Cell Research: Ethic and legal aspects in the supply of surgically removed human tissue for research in the academic and commercial sector in Germany. Cell Tissue Bank 4: 49-56, 2003.

20 Pohl R, Haberl EM, Rein-Fischboeck L, Zimny S, Neumann M, Aslanidis C, Schacherer D, Krautbauer S, Eisinger K, Weiss TS and Buechler C: Hepatic chemerin mRNA expression is reduced in human nonalcoholic steatohepatitis. Eur J Clin Invest 47: 718,2017

21 Matyash V, Liebisch G, Kurzchalia TV, Shevchenko A and Schwudke D: Lipid extraction by methyl-tert-butyl ether for high-throughput lipidomics. J Lipid Res 49: 1137-1146, 2008.

22 Scherer M, Leuthauser-Jaschinski K, Ecker J, Schmitz G and Liebisch G: A rapid and quantitative LC-MS/MS method to profile sphingolipids. J Lipid Res 51: 2001-2011, 2010.

23 Liebisch G, Vizcaino JA, Kofeler H, Trotzmuller M, Griffiths WJ, Schmitz G, Spener F and Wakelam MJ: Shorthand notation for lipid structures derived from mass spectrometry. J Lipid Res 54: 1523-1530, 2013.

24 Breier C, Lisch HJ and Braunsteiner H: Lipoproteins, HDLapolipoproteins, activities of hepatic lipase and lecithincholesterol acyltransferase in the plasma of patients with postalcoholic end-stage liver cirrhosis. Klin Wochenschr 61: 929931, 1983.

25 Wiesner P, Leidl K, Boettcher A, Schmitz G and Liebisch G: Lipid profiling of FPLC-separated lipoprotein fractions by electrospray ionization tandem mass spectrometry. J Lipid Res 50: 574-585, 2009

26 Tacikowski T, Milewski B, Dzieniszewski J, Nowicka G and Walewska-Zielecka B: Comparative analysis of plasma lipoprotein components assessed by ultracentrifugation in primary biliary cirrhosis and chronic hepatitis. Med Sci Monit 6: 325-329, 2000.

27 Li Z, Agellon LB, Allen TM, Umeda M, Jewell L, Mason A and Vance DE: The ratio of phosphatidylcholine to phosphatidylethanolamine influences membrane integrity and steatohepatitis. Cell Metab 3: 321-331, 2006.

28 Nezami Ranjbar MR, Luo Y, Di Poto C, Varghese RS, Ferrarini A, Zhang C, Sarhan NI, Soliman H, Tadesse MG, Ziada DH, Roy R and Ressom HW: GC-MS-based plasma metabolomics for identification of candidate biomarkers for hepatocellular carcinoma in Egyptian cohort. PLoS One 10: e0127299, 2015.

29 He M, Zhang W, Dong Y, Wang L, Fang T, Tang W, Lv B, Chen G, Yang B, Huang P and Xia J: Pro-inflammation NF-kappaB signaling triggers a positive feedback via enhancing cholesterol accumulation in liver cancer cells. J Exp Clin Cancer Res 36: $15,2017$.

30 Feng S, Han M, Zhou L, Wang Q, Li Z, Li Y, Lu H, Liu T, Ma Y, Liu S and Cheng J: NS5ABP37 inhibits liver cancer by impeding lipogenesis and cholesterogenesis. Cancer Sci 108: 1222, 2017.

31 Lee SS, Li J, Tai JN, Ratliff TL, Park K and Cheng JX: Avasimibe encapsulated in human serum albumin blocks cholesterol esterification for selective cancer treatment. ACS Nano 9: 2420-2432, 2015.

32 Chen S, Yin P, Zhao X, Xing W, Hu C, Zhou L and Xu G: Serum lipid profiling of patients with chronic hepatitis B, cirrhosis, and hepatocellular carcinoma by ultra fast LC/IT-TOF MS. Electrophoresis 34: 2848-2856, 2013.

33 Jiang M, Liu F, Xiong WJ, Zhong L, Xu W, Xu F and Liu YB: Combined MELD and blood lipid level in evaluating the prognosis of decompensated cirrhosis. World J Gastroenterol 16: 1397-1401, 2010.

34 Peng K, Mo Z and Tian G: Serum lipid abnormalities and nonalcoholic fatty liver disease in adult males. Am J Med Sci 353: 236-241, 2017. 
35 Shibahara J, Ando S, Sakamoto Y, Kokudo N and Fukayama M: Hepatocellular carcinoma with steatohepatitic features: a clinicopathological study of Japanese patients. Histopathology 64: 951-962, 2014.

36 Ristic-Medic D, Takic M, Vucic V, Kandic D, Kostic N and Glibetic M: Abnormalities in the serum phospholipids fatty acid profile in patients with alcoholic liver cirrhosis - a pilot study. $\mathbf{J}$ Clin Biochem Nutr 53: 49-54, 2013.
37 Wu T, Zheng X, Yang M, Zhao A, Li M, Chen T, Panee J, Jia W and Ji G: Serum lipid alterations identified in chronic hepatitis B, hepatitis B virus-associated cirrhosis and carcinoma patients. Sci Rep 7: 42710, 2017.

Received May 4, 2017

Revised May 16, 2017 Accepted May 19, 2017 